\title{
Personal audio with a planar bright zone
}

\author{
Philip Coleman, ${ }^{\text {a) }}$ Philip J. B. Jackson, and Marek Olik \\ Centre for Vision, Speech and Signal Processing, University of Surrey, Guildford, Surrey, GU2 7XH, \\ United Kingdom \\ Jan Abildgaard Pedersen ${ }^{\text {b) }}$ \\ Bang \& Olufsen A/S, Peter Bangs Vej 15, DK7600, Struer, Denmark
}

(Received 1 April 2014; revised 28 July 2014; accepted 5 August 2014)

\begin{abstract}
Reproduction of multiple sound zones, in which personal audio programs may be consumed without the need for headphones, is an active topic in acoustical signal processing. Many approaches to sound zone reproduction do not consider control of the bright zone phase, which may lead to selfcancellation problems if the loudspeakers surround the zones. Conversely, control of the phase in a least-squares sense comes at a cost of decreased level difference between the zones and frequency range of cancellation. Single-zone approaches have considered plane wave reproduction by focusing the sound energy in to a point in the wavenumber domain. In this article, a planar bright zone is reproduced via planarity control, which constrains the bright zone energy to impinge from a narrow range of angles via projection in to a spatial domain. Simulation results using a circular array surrounding two zones show the method to produce superior contrast to the least-squares approach, and superior planarity to the contrast maximization approach. Practical performance measurements obtained in an acoustically treated room verify the conclusions drawn under free-field conditions.

(C) 2014 Acoustical Society of America. [http://dx.doi.org/10.1121/1.4893909]
\end{abstract}

PACS number(s): 43.60.Fg, 43.60.Pt, 43.60.Tj, 43.38.Hz [NX]

Pages: $1725-1735$

\section{INTRODUCTION}

The topic of personal audio has generated great interest since the $1990 \mathrm{~s},{ }^{1,2}$ and array signal processing to this end is an active problem for sound field control. Sound zones fundamentally require the creation of a spatial cancellation region, and requirements on the target region may additionally be imposed. The development of sound zones in the literature has seen the emergence of techniques which broadly fall in to two categories. ${ }^{3}$ One technique, with its heritage in sound field synthesis (SFS), is to precisely specify a target sound field, creating a dark zone by attenuating the sound pressure over a region. Such control has been considered analytically based on sound field coefficient translation, ${ }^{4}$ which allows the sound zones to be represented as part of a global sound field for control by existing techniques such as wave field synthesis (WFS) ${ }^{5}$ or mode-matching. ${ }^{6}$ The problem of creating a dark zone contained entirely within a bright zone has also been considered analytically. ${ }^{7}$ Alternatively, multi-point pressure matching (PM) optimization ${ }^{8,9}$ attempts directly to minimize the error between desired sound pressures at discretized points and the sound field reproduced by the array. The analytical and least-squares approaches exhibit similar properties in terms of artifacts arising from discretized loudspeaker arrays. ${ }^{3,10}$ Typically, the desired field over a 2-D zone is a plane wave, although any sound field could be specified in principle. In practical personal audio systems, the PM approach has often been adopted ${ }^{11,12}$ as it

\footnotetext{
a) Author to whom correspondence should be addressed. Electronic mail: p.d.coleman@surrey.ac.uk

b) Present address: Dynaudio A/S, Sverigesvej 15, DK8660, Skanderborg, Denmark.
}

generalizes to arbitrary loudspeaker and microphone array geometries and, in principle, includes direct room compensation if measured transfer functions are utilized (although in practice the room response is time-variant).

Alternatively, the energy in the zones can be manipulated. For instance, brightness control ${ }^{13}$ uses constrained optimization to efficiently focus the energy in to a region, and acoustic contrast control (ACC) ${ }^{13}$ additionally creates a level difference between the zones by maximization of the ratio of squared pressures between the zones. ACC has been extensively investigated and implemented, with applications considering personal audio for computer users, ${ }^{14,15}$ aircraft passengers, ${ }^{16,17}$ car passengers, ${ }^{12}$ and users of mobile devices. ${ }^{18-20}$ A similar cost function ${ }^{21}$ maximizes the level difference between the zones to avoid the matrix inversion and allow for adjustment of the array control effort. The performance of this method is bounded by ACC and brightness control depending on the value of a weighting parameter which acts as regularization. ${ }^{19}$

Comparisons of least-squares optimization and energy cancellation approaches under identical conditions s,11,22 $^{3,2}$ found that each technique yielded distinct properties. In particular, although excellent acoustic contrast is produced using energy cancellation, the cost function utilizes spatially summed squared pressures and consequently the phase in the target zone is uncontrolled. Additionally, the perceived audio quality of these approaches has been questioned. ${ }^{11}$ When the loudspeakers surround the zones, as may often be the case in a listening room, highly self-canceling target sound fields may be reproduced due to multiple energy components impinging on the zone from various directions. The SFS approaches are able to resolve these issues by producing planar bright zones, but at the cost of some contrast 
performance and with high array effort. ${ }^{3}$ Furthermore, the minimization of reproduction error rather than contrast leads to a narrower range of frequencies over which a good level of contrast is achieved. ${ }^{3}$

Accordingly, recent attention has been given to using modified least-squares optimization for personal audio, including weighting the optimization ${ }^{23}$ to balance between the zone performance, and using least-squares optimization for the bright zone with alternative constraints to create acoustic contrast. ${ }^{24,25}$ Alternatively, judicious choices of the target field for least-squares optimization have been chosen such that the acoustic contrast and control effort are improved. $^{12}$ One potential limitation of these approaches is that a strict target field is specified, trading the ability of the array to create acoustic contrast against the necessity of approximating the desired pressure vector. While reproduction of specific plane wave components may sometimes be necessary (e.g., for spatial audio), in many cases a less strict definition of the target field may suffice, assuming that desirable zone attributes are retained (such as homogeneity of sound level across the zones and the avoidance of self-cancellation problems). In this regard, achieving high planarity (Sec. II B) may optimize the listening experience without requiring explicit plane wave synthesis. ${ }^{3,26}$

For single-zone reproduction, local approaches to plane wave synthesis have considered the manipulation of intensity $^{27}$ and the focusing of energy in the wavenumber domain. ${ }^{28}$ The latter wavenumber domain point focusing (WDPF) approach does not require an estimate of the pressure gradient in the zone and may therefore be straightforwardly applied to a pressure microphone array, as adopted for existing multi-point sound zone approaches. The planarity control (PC) cost function ${ }^{29}$ is based on a similar concept, using superdirective microphone array beamforming for spatial filtering of the bright zone energy in the context of sound zones. Furthermore, the incoming plane wave direction with respect to the target zone can be specified over a range of angles, rather than a single one. In this way, a planar sound field can be reproduced (alongside excellent cancellation), but the optimization is free to find the best plane wave direction within the specified range.

This article extends the scope of the current literature by fully introducing PC optimization as a means of improving the energy distribution in the target zone where cancellation is also created. New simulation results are presented to explore the cost function performance with a circular array surrounding two zones, and measured performance data are presented, validating the technique in an acoustically treated room, compared to implementations of ACC and PM. The measured performance comparison extends the work of Ref. 3 with practical results in a non-anechoic environment, and also gives some insight into the practical usefulness of sound zone optimization methods applied to circular arrays, complementing recent work applying the techniques in a car cabin $^{12}$ and listening room. ${ }^{30}$

In Sec. II, the background is given, and in Sec. III existing sound field control methods are introduced. The PC cost function is presented in Sec. IV. The performance of the method is demonstrated via simulations in Sec. V and measurements in Sec. VI. The implications are discussed in Sec. VII and the work is briefly summarized in Sec. VIII.

\section{BACKGROUND}

In the following sections, the sound zone notation and evaluation metrics are introduced.

\section{A. Notation}

Figure 1 shows the sound zone system considered throughout this article. Two audio programs A and B are to be reproduced in zones A and B, respectively, and the rest of the room is uncontrolled. The zones (defined acoustically by the control microphone positions) and loudspeakers may be placed arbitrarily in the room. For a single frequency, the complex source weights $q^{l}$ at the $l$ th loudspeaker can be written in vector notation as $\mathbf{q}=\left[q^{1}, q^{2}, \ldots, q^{L}\right]^{T}$, where there are $L$ loudspeakers, and the complex pressures at the control microphone positions are written as $\mathbf{p}_{A}=\left[p_{A}^{1}, p_{A}^{2}, \ldots, p_{A}^{N_{A}}\right]^{T}$ and $\mathbf{p}_{B}=\left[p_{B}^{1}, p_{B}^{2}, \ldots, p_{B}^{N_{B}}\right]^{T}$ for zones $\mathrm{A}$ and $\mathrm{B}$, respectively, where there are $N_{A}$ control microphones in zone A and $N_{B}$ in zone $\mathrm{B}$, and the complex pressures at the $n$th microphones in each zone are $p_{A}^{n}$ and $p_{B}^{n}$. The observed pressures at the monitor microphones are denoted similarly as $\mathbf{o}_{A}=\left[o_{A}^{1}, o_{A}^{2}, \ldots, o_{A}^{M_{A}}\right]^{T}$ and $\mathbf{o}_{B}=\left[o_{B}^{1}, o_{B}^{2}, \ldots, o_{B}^{M_{B}}\right]^{T}$, where there are $M_{A}$ monitor microphones in zone $\mathrm{A}$ and $M_{B}$ in zone $\mathrm{B}$, and the complex pressures at the $m$ th microphones in each zone are $o_{A}^{m}$ and $o_{B}^{m}$. Spatially distinct microphones are used in order to reduce possible bias due to measurement of performance at the exact control positions. The plant matrices contain the transfer functions between each loudspeaker and microphone, and are considered with respect to the control

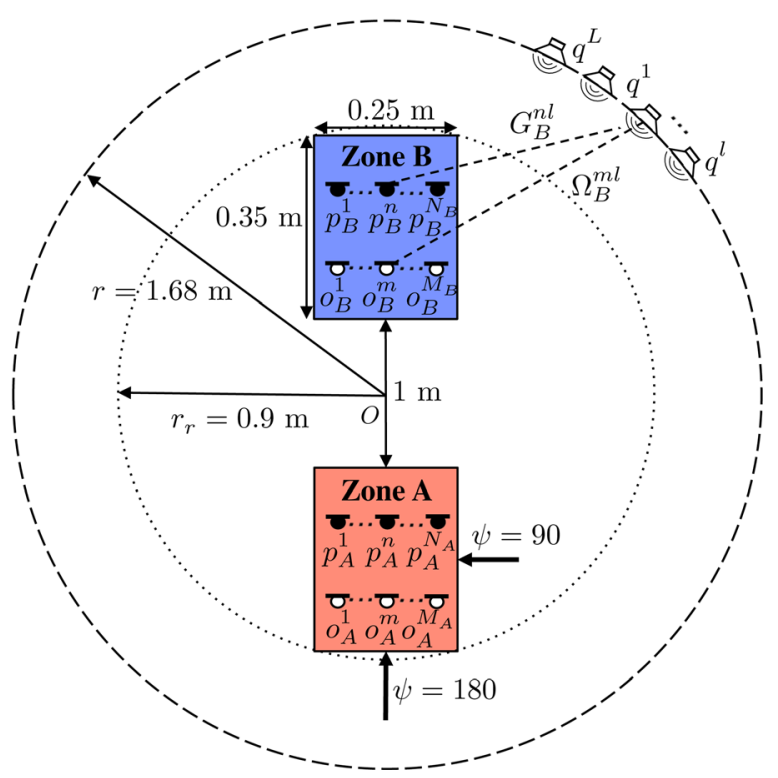

FIG. 1. (Color online) System geometry with $L$ loudspeakers arranged around a radius $r$, and zones $\mathrm{A}$ and $\mathrm{B}$, enclosed by the radius $r_{r}$ and comprising $N_{A}$ and $N_{B}$ control microphones (black) and $M_{A}$ and $M_{B}$ monitor microphones (white), respectively. The transfer functions $G$ and $\Omega$ are also shown. The plane wave directions $\psi$ arriving at zone A are shown. 
and monitor microphones in each zone. For zone A they are defined as

$$
\mathbf{G}_{A}=\left(\begin{array}{ccc}
G_{A}^{11} & \cdots & G_{A}^{1 L} \\
\vdots & \ddots & \vdots \\
G_{A}^{N_{A} 1} & \cdots & G_{A}^{N_{A} L}
\end{array}\right), \quad \boldsymbol{\Omega}_{A}=\left(\begin{array}{ccc}
\Omega_{A}^{11} & \cdots & \Omega_{A}^{1 L} \\
\vdots & \ddots & \vdots \\
\Omega_{A}^{M_{A} 1} & \cdots & \Omega_{A}^{M_{A} L}
\end{array}\right),
$$

where $G_{A}^{n l}$ and $\Omega_{A}^{m l}$ are the transfer functions between the $n$th control microphone and the $m$ th monitor microphone in zone $A$, respectively, and the $l$ th loudspeaker. The equivalent notation is used for $\mathbf{G}_{B}$ and $\Omega_{B}$. The pressures at the microphone positions may be written as $\mathbf{p}_{A}=\mathbf{G}_{A} \mathbf{q}, \mathbf{o}_{A}=\boldsymbol{\Omega}_{A} \mathbf{q}$, $\mathbf{p}_{B}=\mathbf{G}_{B} \mathbf{q}$ and $\mathbf{o}_{B}=\mathbf{\Omega}_{B} \mathbf{q}$.

\section{B. Evaluation}

The evaluation metrics of acoustic contrast, control effort and planarity are used to compare the performance of the optimization approaches. ${ }^{3}$ Acoustic contrast describes the attenuation achieved between the zones. The contrast between bright zone $\mathrm{A}$ and dark zone $\mathrm{B}$, which here has a maximum of $76 \mathrm{~dB}$ (see Sec. V), is the ratio of spatially averaged pressures in each zone due to the reproduction of program A, expressed in decibels:

$$
\text { Contrast }=10 \log _{10}\left(\frac{M_{B} \mathbf{o}_{A}^{H} \mathbf{o}_{A}}{M_{A} \mathbf{o}_{B}^{H} \mathbf{o}_{B}}\right) .
$$

The energy that the loudspeaker array requires in order to achieve the reproduced sound field is also an important consideration. A high control effort implies poor acoustical efficiency, with high sound pressure levels (SPLs) emitted in to the room, and is also linked to decreased robustness to errors ${ }^{3}$ and the physical realizibility of a set of control filters. The control effort describes the total array energy relative to a single reference source $q_{r}$ producing the same pressure in the bright zone and is expressed in decibels as

$$
\text { Effort }=10 \log _{10}\left(\frac{\mathbf{q}^{H} \mathbf{q}}{\left|q_{r}\right|^{2}}\right) .
$$

Planarity is a measure of the proportion of the energy in the bright zone that can be attributed to the direction of the principal energy component, ${ }^{26}$ and is well suited to the situation considered here as it provides an objective measure of the spatial sound field properties without requiring a target field to be strictly defined. The energy distribution at the microphone array (over incoming plane wave direction) is given by $w_{i}=(1 / 2)\left|\psi_{i}\right|^{2}$, where $\mathbf{w}=\left[w_{1}, w_{2}, \ldots\right.$, $\left.w_{I}\right]^{T}$ are the energy components at the $i$ th angle (the angular spectrum) and $\psi_{i}$ is the plane wave component at the $i$ th angle. The steering matrix $\mathbf{H}_{A}$ of dimensions $I \times M_{A}$ maps between the observed pressures at the microphones and the plane wave components and is defined such that

$$
\mathbf{w}=\frac{1}{2}\left|\mathbf{H}_{A} \mathbf{o}_{A}\right|^{2}
$$

The elements of $\mathbf{H}_{A}$ are here populated by a regularized max-SNR beamformer ${ }^{31}$ with fixed beamwidth ${ }^{3,26,29}$ (i.e., an ACC beamformer). This has superdirective properties with respect to the classical delay-and-sum beamformer (which is equivalent to the spatial Fourier transform). ${ }^{26}$ The planarity metric is defined as the ratio between the energy due to the largest plane wave component and the total energy flux of plane wave components:

$$
\text { Planarity }=\frac{\sum_{i} w_{i} \mathbf{u}_{i} \cdot \mathbf{u}_{\alpha}}{\sum_{i} w_{i}},
$$

where $\mathbf{u}_{i}$ is the unit vector associated with the $i$ th component's direction, $\mathbf{u}_{\alpha}$ is the unit vector in the direction $\alpha=\arg$ $\max _{i} w_{i}$, and $\cdot$ denotes the inner product. Where a plane wave is reproduced, the planarity score approaches $100 \%$, and the score is exactly $0 \%$ for highly self-canceling sound fields. The planarity score for a diffuse sound field also tends toward $0 \%$ (as the long-term average angular energy spectrum tends toward uniform), but repeated short term measurements vary over time and frequency due to the random nature of a diffuse field. A planarity of zero cannot therefore directly discern between these kinds of sound fields. However, the angular spectrum $\mathbf{w}$ can distinguish between a standing wave (equal and opposite peaks) and a uniform distribution (a noisy approximation of which is measured by each diffuse-field snapshot). We assume that the propagating portion of the wave dominates the wave field at the measurement positions for the frequencies of interest.

\section{THEORY}

In the following sections, ACC, PM, and WDPF optimizations are introduced. These utilize constraints on the sum of squared pressures in zone $\mathrm{A}$ and the sum of squared source weights. The former can be expressed as $A=N_{A}\left|p_{r}\right|^{2}$ $\times 10^{T / 10}$, where $T$ is the target spatially averaged level in decibels relative to the threshold of hearing $p_{r}=20 \mu \mathrm{Pa}$, and the latter as $Q=\left|q_{r}\right|^{2} \times 10^{E / 10}$ [cf. Eq. (3)].

\section{A. Acoustic contrast control}

ACC maximizes the ratio of the spatially averaged sound pressures between the bright zone and the dark zone. Introducing the "indirect" formulation, ${ }^{19}$ the cost function may be written as a minimization of the pressure in the dark zone, with constraints imposed on both $A$ and $Q$ (Refs. 13 and 19):

$$
J=\mathbf{p}_{B}^{H} \mathbf{p}_{B}+\mu\left(\mathbf{p}_{A}^{H} \mathbf{p}_{A}-A\right)+\lambda\left(\mathbf{q}^{H} \mathbf{q}-Q\right),
$$

where $H$ denotes the Hermitian transpose. The cost function may be minimized by setting the derivatives with respect to $\mathbf{q}$ and the Lagrange multipliers $\mu$ and $\lambda$ to zero: 
$-\left(\mathbf{G}_{A}^{H} \mathbf{G}_{A}\right)^{-1}\left(\mathbf{G}_{B}^{H} \mathbf{G}_{B}+\lambda \mathbf{I}\right) \mathbf{q}=\mu \mathbf{q} ; \quad \mathbf{p}_{A}^{H} \mathbf{p}_{A}=A ; \quad \mathbf{q}^{H} \mathbf{q}=Q$,

where $\mathbf{I}$ is the identity matrix and $\mathbf{q}$ is proportional to the eigenvector $\hat{\mathrm{q}}$ corresponding to the maximum eigenvalue of $\left(\mathbf{G}_{B}^{H} \mathbf{G}_{B}+\lambda \mathbf{I}\right)^{-1}\left(\mathbf{G}_{A}^{H} \mathbf{G}_{A}\right) .{ }^{19}$ The constraint that $A$ equals a certain fixed value is enforced by scaling $\hat{\mathrm{q}}$, and the second Lagrange multiplier $\lambda$ must be chosen such that the effort constraint is satisfied. If $Q>\mathbf{q}^{H} \mathbf{q}$ when $\lambda=0$, the constraint is not active. When $\lambda>0$, it acts as regularization by trading the control effort for increased bright zone energy and improving the numerical condition of the inversion of $\mathbf{G}_{B}^{H} \mathbf{G}_{B}$. In our implementation, $\lambda$ is first initialized ${ }^{3}$ such that the condition number of $\left(\mathbf{G}_{B}^{H} \mathbf{G}_{B}+\lambda \mathbf{I}\right)$ does not exceed $10^{10}$, then determined numerically using a gradient descent search such that $Q \geq \mathbf{q}^{H} \mathbf{q}$ when $A$ has been fixed.

\section{B. Pressure matching}

Under the least-squares approach, the error between the desired and reproduced sound fields is minimized. A plane wave sound field can be written as $\mathbf{d}_{A}=D_{A} e^{j k \mathbf{r}_{n} \cdot \mathbf{u}_{\varphi}}$, for $n=1$, $2, \ldots, N_{A}$, where $D_{A}$ gives the pressure amplitude, $\mathbf{r}_{n}$ is the position of the $n$th control microphone in zone $\mathrm{A}$, · denotes the inner product, and $\mathbf{u}_{\varphi}$ is the unit vector in the direction of the incoming plane wave. The desired zone B sound field is given by a vector of length $N_{B}$ populated with zeros, $\mathbf{d}_{B}=\mathbf{0}$. The cost function, constrained to a sum of squared source weights $Q$, is ${ }^{8}$

$$
J=\left(\mathbf{p}_{A}-\mathbf{d}_{A}\right)^{H}\left(\mathbf{p}_{A}-\mathbf{d}_{A}\right)+\mathbf{p}_{B}^{H} \mathbf{p}_{B}+\lambda\left(\mathbf{q}^{H} \mathbf{q}-Q\right) .
$$

Using the method of Lagrange multipliers the solution can be found by taking the derivatives with respect to $\mathbf{q}$ and $\lambda$ :

$$
\mathbf{q}=\left(\mathbf{G}_{A}^{H} \mathbf{G}_{A}+\mathbf{G}_{B}^{H} \mathbf{G}_{B}+\lambda \mathbf{I}\right)^{-1} \mathbf{G}_{A}^{H} \mathbf{d}_{A} ; \mathbf{q}^{H} \mathbf{q}=Q .
$$

The Lagrange multiplier $\lambda$ is numerically chosen to satisfy the control effort constraint, and it is assumed that the solution is appropriately scaled by setting $\mathbf{d}_{A}^{H} \mathbf{d}_{A}=A$. As for ACC, $\lambda$ is initialized such that the condition number of $\left(\mathbf{G}_{A}^{H} \mathbf{G}_{A}+\mathbf{G}_{B}^{H} \mathbf{G}_{B}+\lambda \mathbf{I}\right)$ does not exceed $1 \times 10^{10}$.

\section{Wavenumber domain point focusing}

The WDPF concept (Ref. 28) exploits the appearance of a plane wave as a point in the wavenumber domain. Existing energy focusing techniques can successfully concentrate sound energy to a spatially confined region. Therefore, by transforming the sound pressures in to the wavenumber domain, the sound energy may be focused toward a point corresponding to a plane wave source. WDPF has been compared over a 2-D zone with implementations of higher-order ambisonics (HOA) using a circular array and WFS with a planar array, and was found to improve accuracy of plane wave reproduction with respect to HOA and require fewer loudspeakers than WFS. ${ }^{32}$

To illustrate the concept and provide the necessary background for the introduction of $\mathrm{PC}$, the problem may be written in a familiar form. A steering matrix $\mathbf{Y}_{A}$ of dimensions $I \times N_{A}$ (designed as $\mathbf{H}_{A}$ using the monitor microphone positions), projects the sound pressure at the control microphones in to the angular spectrum, mapping between the complex pressures at the microphones and the reproduced plane wave energy distribution over azimuth. For planar sound field reproduction in a single (bright) zone, an appropriate cost function is to maximize the brightness via such a spatial domain:

$$
J=\mathbf{p}_{A}^{H} \mathbf{Y}_{A}^{H} \boldsymbol{\Gamma} \mathbf{Y}_{A} \mathbf{p}_{A}-\lambda\left(\mathbf{q}^{H} \mathbf{q}-Q\right),
$$

which closely resembles the brightness control cost function. ${ }^{13}$ Thus, Eq. (10) can be interpreted as the maximization of acoustic brightness via the spatial domain, constrained by $Q$ to a certain sum of squared source weights. The term $\Gamma$ is a diagonal matrix allowing a weighting to be applied based on the desired incoming plane wave directions:

$$
\boldsymbol{\Gamma}=\operatorname{diag}\left[\gamma_{1}, \gamma_{2}, \ldots, \gamma_{I}\right]
$$

where $0 \leq \gamma_{i} \leq 1$ is the weighting corresponding to the $i$ th steering angle. Energy will therefore be focused in the direction of the nonzero elements of $\boldsymbol{\Gamma}$.

Although WDPF was introduced ${ }^{28}$ as a generalized 3-D technique using a spherical harmonics representation of the sound field, it can be expressed by Eq. (10), with $\mathbf{Y}_{A}$ populated by spatial Fourier transform and the diagonal of $\boldsymbol{\Gamma}$ as a Kronecker delta (unit impulse) at the desired plane wave direction $\gamma_{\varphi}$.

\section{PLANARITY CONTROL}

The WDPF approach provides an interesting perspective on the problem of plane wave reproduction over a zone, and inspection of Eqs. (6) and (10) shows a clear opportunity to introduce such spatial filtering to the ACC sound zone optimization. The PC optimization cost function can thus be introduced as a minimization of the dark zone pressures (as ACC) with the bright zone energy constraint enforced via the spatial domain (as WDPF), and with an effort constraint:

$$
J=\mathbf{p}_{B}^{H} \mathbf{p}_{B}+\mu\left(\mathbf{p}_{A}^{H} \mathbf{Y}_{A}^{H} \boldsymbol{\Gamma} \mathbf{Y}_{A} \mathbf{p}_{A}-A\right)+\lambda\left(\mathbf{q}^{H} \mathbf{q}-Q\right) .
$$

As in Eq. (6), $\mu$ and $\lambda$ are Lagrange multipliers. The solution is found, as for ACC, by setting to zero the derivatives with respect to $\mathbf{q}$ and each of the Lagrange multipliers:

$$
\begin{gathered}
-\left(\mathbf{G}_{A}^{H} \mathbf{Y}_{A}^{H} \boldsymbol{\Gamma} \mathbf{Y}_{A} \mathbf{G}_{A}\right)^{-1}\left(\mathbf{G}_{B}^{H} \mathbf{G}_{B} \mathbf{q}+\lambda \mathbf{q}\right)=\mu \mathbf{q} ; \\
\mathbf{p}_{A}^{H} \mathbf{Y}_{A}^{H} \boldsymbol{\Gamma} \mathbf{Y}_{A} \mathbf{p}_{A}=A ; \mathbf{q}^{H} \mathbf{q}=Q .
\end{gathered}
$$

The optimal source weights are proportional to the eigenvector corresponding to the maximum eigenvalue of $\left(\mathbf{G}_{B}^{H} \mathbf{G}_{B}+\lambda \mathbf{I}\right)^{-1}\left(\mathbf{G}_{A}^{H} \mathbf{Y}_{A}^{H} \boldsymbol{\Gamma} \mathbf{Y}_{A} \mathbf{G}_{A}\right)$. The values of the Lagrange multipliers are determined iteratively as above, where the sum of squared pressures (projected via the angular spectrum) is fixed to satisfy the constraint $A=\mathbf{p}_{A}^{H} \mathbf{Y}_{A}^{H} \boldsymbol{\Gamma} \mathbf{Y}_{A} \mathbf{p}_{A}$, and $\lambda$ is initialized based on the matrix condition number and chosen such that the constraint on $\mathbf{q}^{H} \mathbf{q}$ is satisfied.

The design of $\boldsymbol{\Gamma}$, with weightings $\gamma$ between zero and one, is clearly a significant factor in $\mathrm{PC}$ implementation. If the diagonal is filled with ones, then PC is identical to ACC [Eq. (6)]. If, on the other hand, the vector is populated with 


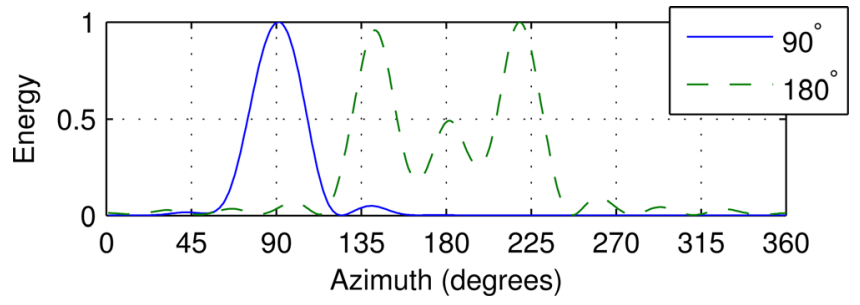

FIG. 2. (Color online) Energy distribution over azimuth for $\boldsymbol{\Gamma}$ set as a narrow window around $90^{\circ}$ (solid) and $180^{\circ}$ (dashed), simulated at $1 \mathrm{kHz}$ using the geometry in Fig. 1.

zeros apart from a single target direction, a plane wave impinging from the specified direction should be reproduced, similar to WDPF. Expression of the mapping in to the spatial domain presents an opportunity to set a range of pass angles rather than a single plane wave direction (corresponding to focusing the energy toward an arc on the radiation circle in the wavenumber domain, rather than a single point). For monophonic sound zones, the primary motivation for a planar bright zone is to restrict the plane wave components such that self-cancellation patterns (e.g., energy notches) do not occur across the bright zone. Therefore, the actual angle of the source may not need to be strictly specified to create a planar bright zone. However, if required for plane wave reproduction (such as for spatial audio in sound zones), fewer non-zero elements of $\boldsymbol{\Gamma}$ may be used. In Fig. 2, viable designs of $\boldsymbol{\Gamma}$ for two-zone reproduction are illustrated at $1 \mathrm{kHz}$, based on anechoic simulations of the geometry in Fig. 1. The diagonal elements $\gamma_{i}$ were set with $\gamma_{\varphi}=1$ and a raised-cosine weighting for $i=\varphi \pm 5$, with $\varphi$ set at $90^{\circ}$ and $180^{\circ}$. For the $90^{\circ}$ angle, a planar sound field with high contrast was reproduced ( $96 \%$ planarity, $76 \mathrm{~dB}$ contrast), with the energy centered at the expected location. At $180^{\circ}$ (where the sound energy would need to be transmitted across the dark zone), the contrast was also $76 \mathrm{~dB}$, but the planarity dropped to $60 \%$. These cases illustrate that PC can generally reproduce a planar sound field within a small angular pass range, but where such a range dictates that energy propagates across the dark zone, acoustic contrast is prioritized. Such behavior follows from the fundamental minimization of dark zone pressure in Eq. (12). In the following sections, simulation results and measured performance data are presented to demonstrate the performance of PC with a wider angular pass range.

\section{SIMULATIONS}

In this section, the performance of $\mathrm{PC}$ is demonstrated by means of anechoic simulations. A 60 channel circular array of equally spaced loudspeakers around a radius of 1.68 $\mathrm{m}$ was adopted, illustrated in Fig. 1. Control and monitor microphones in the zones were spaced $2.5 \mathrm{~cm}$ apart, fulfilling the Nyquist spatial sampling criterion up to $6.8 \mathrm{kHz}$. In each case there were 192 omnidirectional microphones in each zone, arranged to sample a $25 \mathrm{~cm} \times 35 \mathrm{~cm}$ grid. The zones were $1 \mathrm{~m}$ apart at the closest edges, and enclosed by a reproduction radius $r_{r}$ of $0.9 \mathrm{~m}$. Monitor microphones outside of the zones, used only to render visualizations of the sound
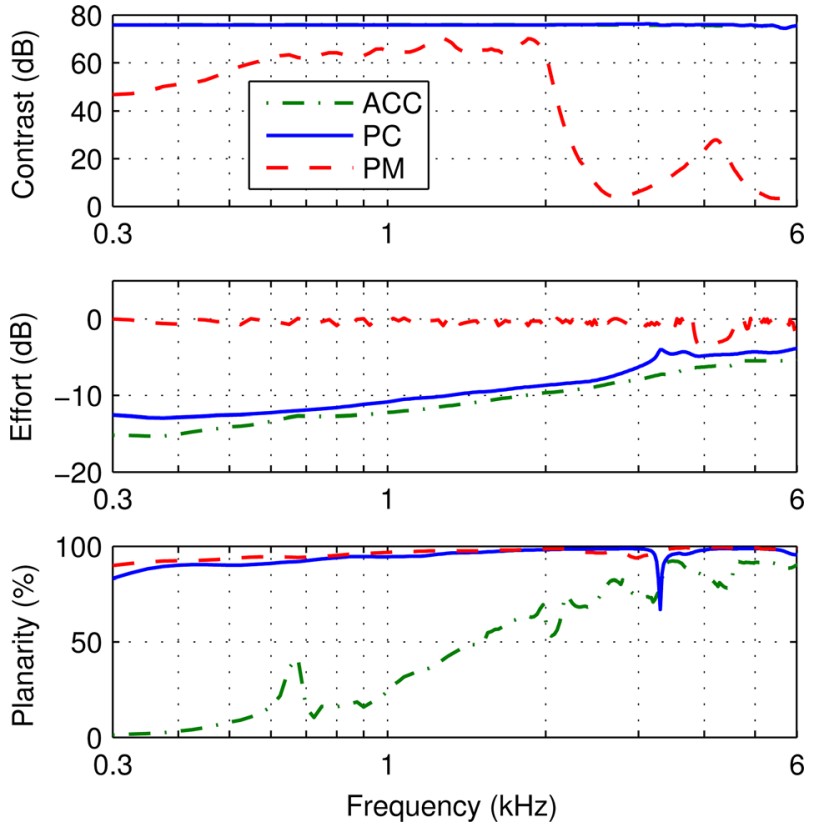

FIG. 3. (Color online) Simulated performance of ACC (dot-dash), PC (solid) and PM (dashed) under the metrics of contrast (top), effort (middle), and planarity (bottom).

field, were spaced at $10 \mathrm{~cm}$. The free-field Green's function was used to populate the plant matrices,

$$
G^{n l}=\frac{j \rho c k}{4 \pi R} e^{j k R}, R=\left|\mathbf{x}^{n l}\right|,
$$

where $\rho=1.21 \mathrm{~kg} / \mathrm{m}^{3}, c=343 \mathrm{~m} / \mathrm{s}, k$ is the wavenumber $\omega / c$, and $\mathbf{x}^{n l}$ is the relative position vector between the $n$th microphone and the $l$ th loudspeaker. The frequencydependent regularization parameter was selected for each method by initializing $\lambda$ (as discussed above) and further enforcing a $0 \mathrm{~dB}$ control effort limit if necessary. ${ }^{3}$ For all methods, the target level was set at $T=76 \mathrm{~dB}$ SPL, which has been shown to be a comfortable listening level and has been used during listening tests based on the sound zone interference situation. ${ }^{33}$ Pressures below $0 \mathrm{~dB}$ SPL are not considered, which imposes an upper limit on the maximum contrast. ${ }^{3}$ The range of $\boldsymbol{\Gamma}$ was set to allow a wide range of incoming energy directions between $30^{\circ}$ and $150^{\circ},{ }^{29}$ and the plane wave direction for PM was set at $90^{\circ}$.

The simulation results are shown in Fig. 3. The PC contrast performance was very good and consistent across the midrange frequency band of $300-6000 \mathrm{~Hz}$. The fundamental focus of the cost function [Eq. (12)] is the cancellation term, which is unchanged from that in the ACC cost function [Eq. (6)]. The ACC and PC contrast therefore reached the maximum level of $76 \mathrm{~dB}$ across the frequency range considered, and both methods outperformed PM at all frequencies. The limitations of PM in terms of the bandwidth imposed by the loudspeaker spatial aliasing limit $(1800 \mathrm{~Hz})$ are evident in the contrast behavior. These were alleviated in PC by allowing a larger range of possible pass angles. This advantage is particularly pronounced between 2 and $6 \mathrm{kHz}$.

The issue of the upper frequency bound of ACC and PM has previously been investigated (see Fig. 5 in Ref. 3). In 

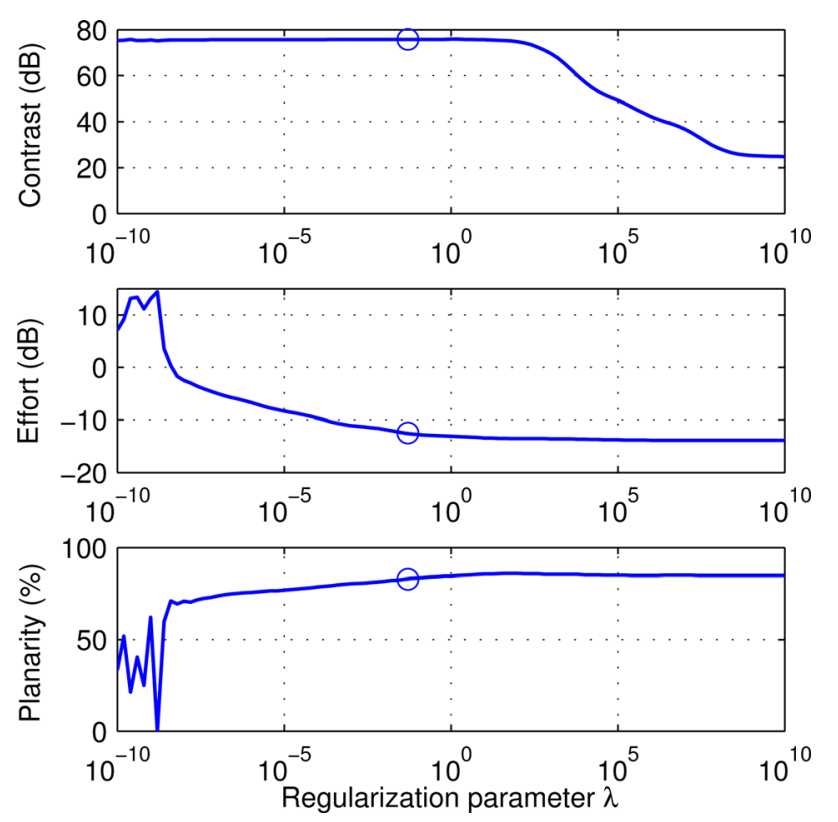

FIG. 4. (Color online) Performance of PC with varied regularization parameter $\lambda$ under ideal conditions, at $300 \mathrm{~Hz}$. The $\lambda$ used for the results plotted in Fig. 3 are indicated $(O)$.

order to compare $\mathrm{PC}$, it is useful to consider the gradient of additional contrast bandwidth with increasing numbers of (equally spaced) loudspeakers $L$. Comparing the frequencies at which the contrast fell $3 \mathrm{~dB}$ below the maximum level, ${ }^{3}$ ACC and PC had bandwidths of $123 \mathrm{~L} \mathrm{~Hz}$ and $120 \mathrm{~L} \mathrm{~Hz}$, respectively, compared to a theoretical value of $127 \mathrm{~L} \mathrm{~Hz}$ based on the projected loudspeaker spacing around the dark zone. ${ }^{3}$ On the other hand, PM had an observed bandwidth of $30 \mathrm{~L} \mathrm{~Hz}$ for this geometry, which closely matched the theoretical value based on the projected loudspeaker spacing around the reproduction radius $r_{r}$. Thus, when a fairly broad angular pass range $\Gamma$ is specified, PC produces greater contrast than PM over a wide bandwidth, and the effect is consistent over various sizes of loudspeaker array.

The PC control effort performance also tended toward that of ACC (which was marginally better over the considered frequency range). Enforcing the maximum matrix condition number of $10^{10}$ was adequate to ensure that the effort fell below $0 \mathrm{~dB}$ for much of the frequency range, and the effort performance was consistently preferable to PM, where it is evident that the $0 \mathrm{~dB}$ limit was enforced over much of the frequency range.

Finally, PC had good planarity performance across frequency, both above and below the $1800 \mathrm{~Hz}$ spatial aliasing limit. Under this metric, the least-squares optimization of PM produced the best scores (synthesizing a plane wave). The narrow notch in PC planarity at $3.3 \mathrm{kHz}$ corresponds to the transition between one and two grating lobes (due to loudspeaker spacing) being present across the sound field. Yet, the PC planarity scores were for the most part similar to PM, and greatly improved from ACC. Thus, the constraint on the energy flux in the bright zone has reduced the appearance of self-cancellation artifacts from the reproduced sound field.

The regularization effect on PC is illustrated at $300 \mathrm{~Hz}$ in Fig. 4, which shows the contrast, effort and planarity

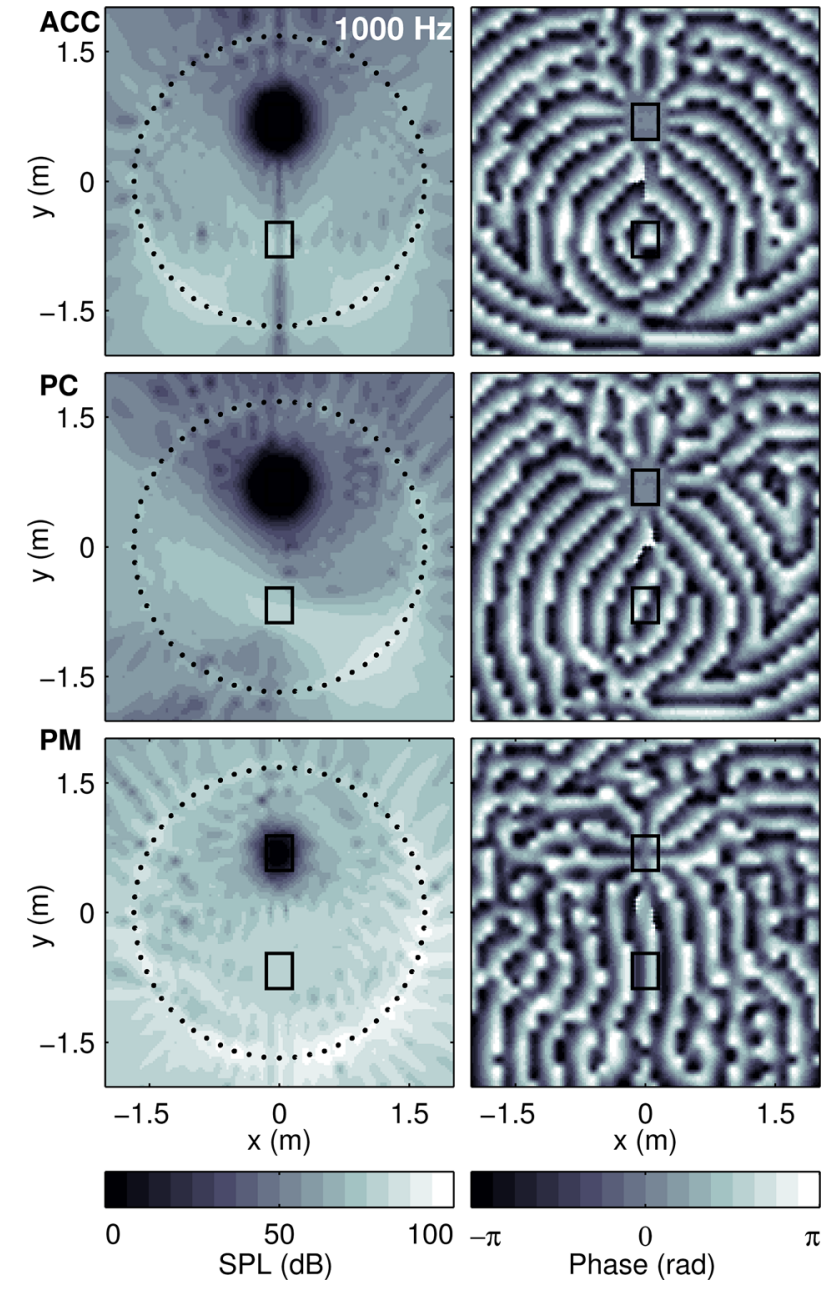

FIG. 5. (Color online) Simulated sound pressure level (left column) and phase (right) distribution of reproduced sound field at $1 \mathrm{kHz}$ using ACC (top row), PC (middle), and PM (bottom).

performance for various values of $\lambda$, directly applied to Eq. (13). The fluctuations in control effort and planarity for very small values of $\lambda$ indicate numerical instability in the results. Therefore, Fig. 4 shows appropriate regularization to be vital for achieving the desired properties from PC. The value of $\lambda$ used in Fig. 3 is marked $(\bigcirc)$ against each curve, and represents a suitable value under each metric. The performance of PC with varied regularization under simulated error conditions is similar to that of ACC. ${ }^{3}$

The contrast, effort and planarity performance obtained using PC can be further clarified by studying the SPL and phase maps, which are shown at $1 \mathrm{kHz}$ in Fig. 5. The standing wave characteristics of ACC are evident from the SPL distribution (top-left), from which two beams can be noted to radiate in to the bright zone, creating a notch along the $y$ axis. The plane wave field (traveling east-west) reproduced by PM can be readily identified from the phase plot (bottom-right), and the increased brightness in the SPL distribution (bottom-left) indicates the higher control effort. By inspection, PC (middle row) can be noted to produce an ACC-like dark zone, yet with a simple beam pattern across the bright zone.

The PC performance over frequency is also clarified by inspection of SPL distributions, shown in Fig. 6 at $300 \mathrm{~Hz}$ 

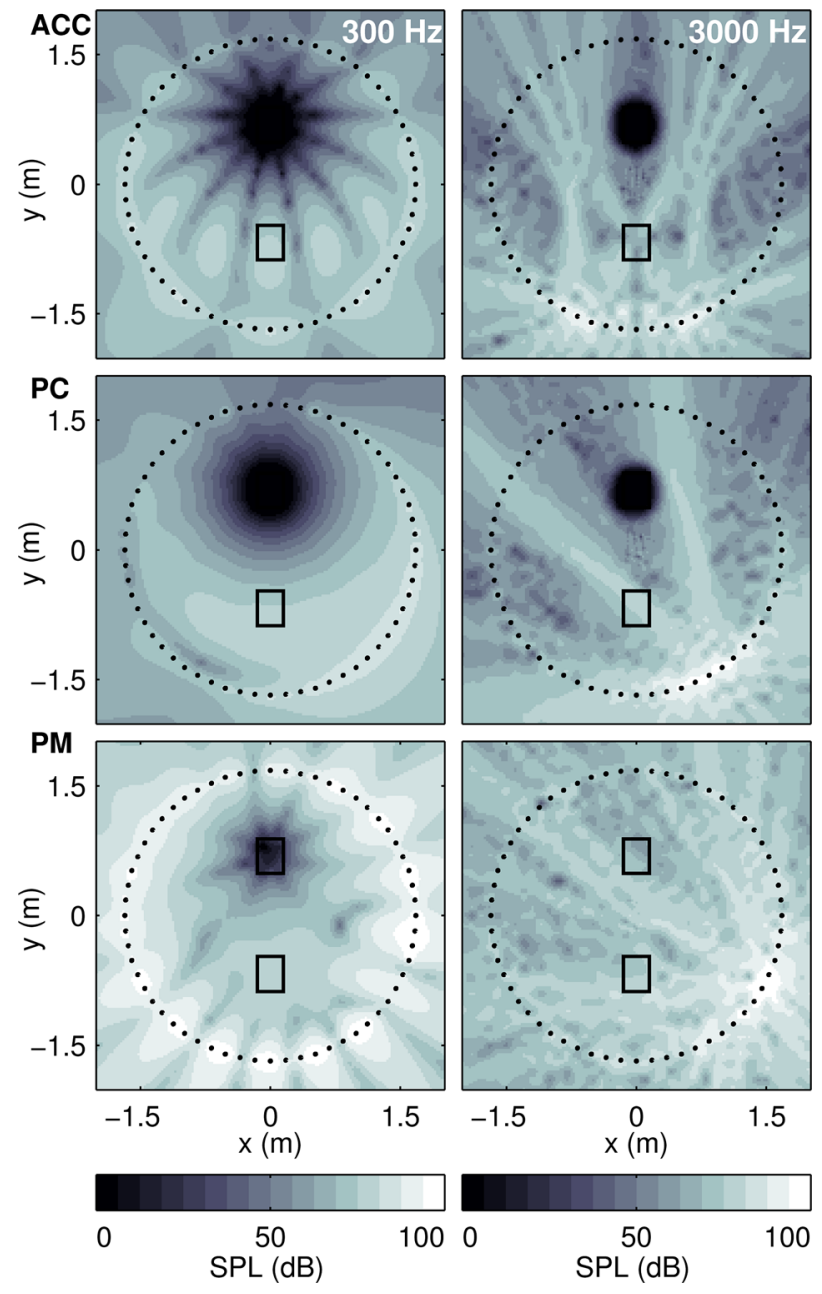

FIG. 6. (Color online) Simulated sound pressure level distribution at $300 \mathrm{~Hz}$ (left column) and $3 \mathrm{kHz}$ (right) using ACC (top row), PC (middle), and PM (bottom).

and $3 \mathrm{kHz}$. From these plots, it can be observed that ACC's self-canceling behavior produces beams from multiple directions, changing with frequency. At $300 \mathrm{~Hz}$, the main energy lobes are widely spaced, and at $3 \mathrm{kHz}$ the interference pattern creating the high level of contrast is rather complex. PC produces a simple bright zone energy pattern at both frequencies, and at $3 \mathrm{kHz}$ the freedom in directional placement allows the grating lobe to be steered around the dark zone while creating a planar bright zone. Similarly, the PM performance trends generalize well across frequency. There is high effort evident at both $300 \mathrm{~Hz}$ and $3 \mathrm{kHz}$, and the dark zone is small even at the lower frequency. At $3 \mathrm{kHz}$, the poor contrast score is explained by the positioning of the grating lobe which appears across the dark zone.

An interesting perspective on the comparative method performance can be gained by analyzing the distribution of energy in the bright zone with respect to azimuth. The planarity scores (Fig. 3, bottom) and the phase distributions in the enclosure (Fig. 5, right) support the case that PC is capable of creating highly planar fields in the target zone, at individual frequencies. However, it is also interesting to observe the range of incoming plane wave directions as a function of frequency. Figure 7 shows the normalized distribution of
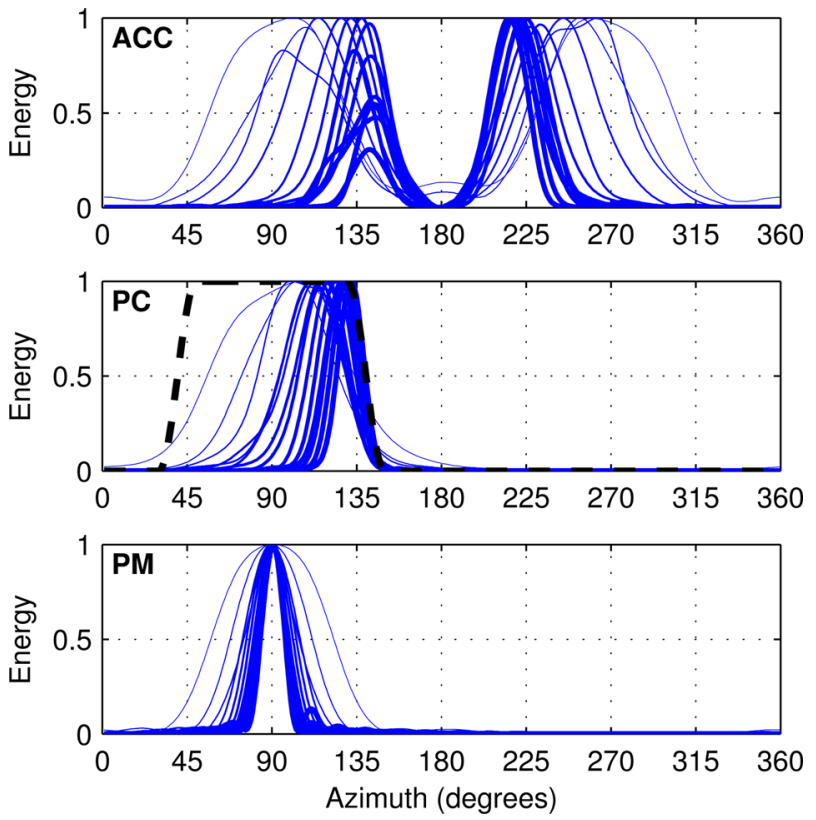

FIG. 7. (Color online) Simulated energy across azimuth, analyzed using the planarity beamformer, for ACC (top), PC (middle), and PM (bottom). The thick dashed line in the middle plot indicates the specified window along the diagonal of $\boldsymbol{\Gamma}$. Each line represents a single frequency, with lines at $200 \mathrm{~Hz}$ intervals between 300 and $3000 \mathrm{~Hz}$ superimposed on each plot. Line thickness increases with frequency.

energy for multiple frequencies between 300 and $3000 \mathrm{~Hz}$, plotted across azimuth for ACC, PC, and PM. In the top plot, the ACC energy can be seen to impinge fairly equally from angles evenly spaced about $180^{\circ}$ (becoming more closely spaced with increasing frequency), which relates well to the energy pattern and corresponding null visible in Fig. 5 (topleft). In contrast for PM, in Fig. 7 (bottom), the energy impinges from the specified direction of $90^{\circ}$ for each frequency plotted.

The energy impinging on the target zone for PC can be seen to conform to the window specified by $\Gamma$ [cf. Eq. (11), drawn as a thick dashed line], with a number of components clustered around $115^{\circ}$. The perceptual effect of such an energy distribution deserves further investigation, but the freedom that the optimization has to place the energy at each frequency has a clear beneficial effect on the achieved contrast between PM and PC, while maintaining a planar energy distribution at individual frequencies and eradicating the self-cancellation of ACC. From our experience and informal listening tests, the self-canceling bright zone energy pattern reproduced by ACC is perceptually undesirable, which may be attributed to instability of source locations in the perceived sound scene. Assuming that poor contrast is also undesirable, PC gives a good balance of characteristics among the state of the art methods.

\section{EXPERIMENTAL VALIDATION}

The anechoic simulations described above indicate that PC is a promising optimization method for sound zones. However, good practical performance is necessary to make significant claims in terms of real-world performance. The performance was therefore evaluated using an experimental 


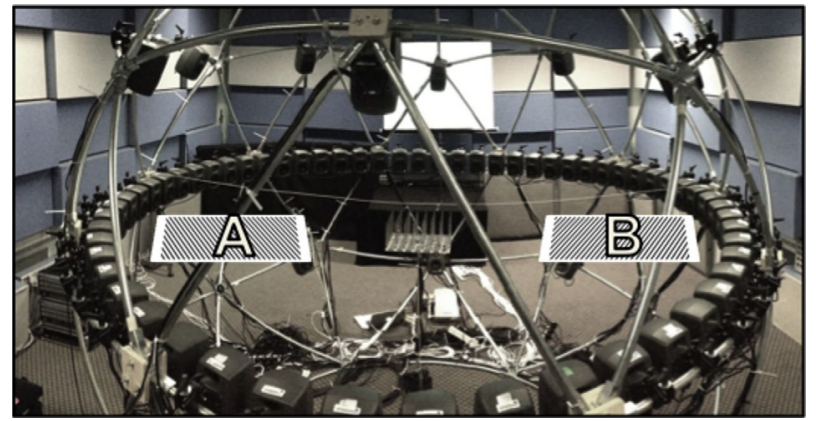

FIG. 8. (Color online) Photograph of the experimental system, with the approximate zone positions marked.

system. The parameters and constraints for each method were specified identically to those in the simulations. In this section, the measurement system is described and measured performance data are presented and analyzed.

\section{A. Reproduction system realization}

A reproduction and measurement system was designed and mounted on a bespoke spherical structure, the "Surrey Sound Sphere" placed in an acoustically treated room of dimensions $6.55 \times 8.78 \times 4.02 \mathrm{~m}$ (RT60 $235 \mathrm{~ms}$ averaged over $0.5,1$, and $2 \mathrm{kHz}$ octave bands). The loudspeakers (Genelec 8020 b) were clamped to the equator of the sphere to form a 60 channel circular array (as Fig. 1), and 48 microphones (Countryman B3 omni) were attached to a grid mounted on a microphone stand. In order to achieve the required sampling density of microphone locations, eight positions of the microphone stand were measured per zone. Alternate microphones in the grid were assigned to be used as control or monitor microphones (see Sec. II A). A photograph of the equipment is shown in Fig. 8. A computer running Matlab was used to play the audio and also to record the signals from the microphones, via the "playrec" utility. A 72 channel MOTU PCIe 424 sound card was used for the analog to digital interface, with the microphone inputs first passed through a pre-amplifier stage (PreSonus Digimax D8). Level differences between the input and output signal channels were compensated through calibration. Room impulse responses between each microphone position and each loudspeaker were measured using the maximum length sequence (MLS) approach (15th order at $48 \mathrm{kHz}$ ) and cropped at $150 \mathrm{~ms}$ after the impulse onset.

Finite impulse response (FIR) filters were then populated offline by considering a bin-by-bin approach. The room impulse responses (RIRs) were first down-sampled to the simulation sample rate of $20 \mathrm{kHz}$, and a 8192 point fast Fourier transform (FFT) was taken. The source weights were collated for each frequency bin, the negative frequency bins populated by complex conjugation, and the inverse FFT taken to obtain a time-domain filter. A 4096 sample modeling delay was applied to ensure causality. Independent measurements of objective performance were made by repositioning the microphone array in the desired monitor location, simultaneously replaying an MLS sequence convolved with each of the FIR control filters, and sampling the reproduced sound pressures with the microphone array.
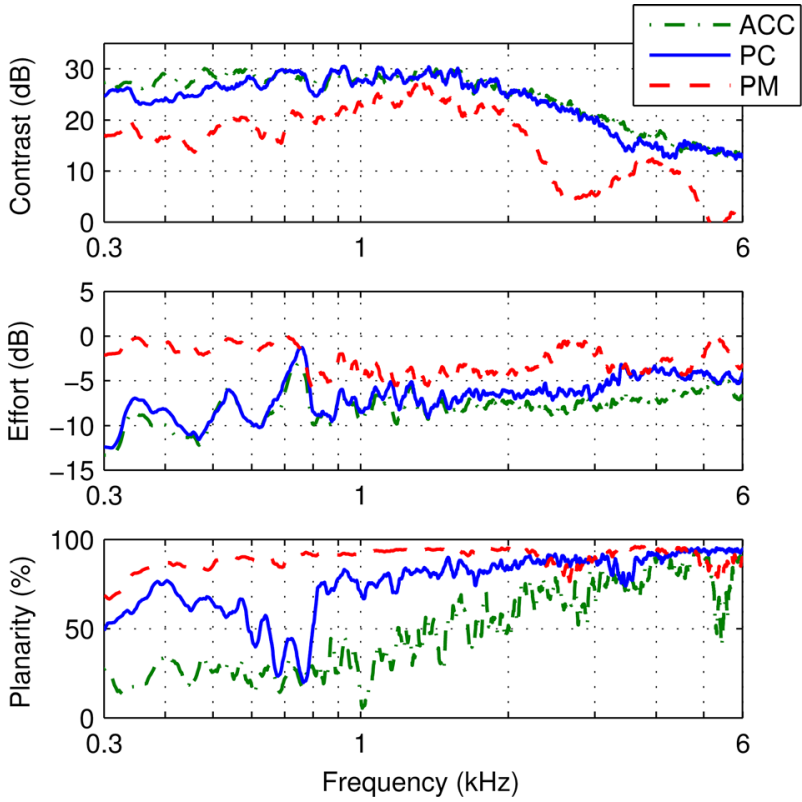

FIG. 9. (Color online) Measured performance of ACC (dot-dash), PC (solid) and PM (dashed) under the metrics of contrast (top), effort (middle) and planarity (bottom).

Finally, the FFT was taken of the measured system responses, and the evaluation metrics were calculated in the frequency domain.

\section{B. Performance}

As above, the baseline methods of ACC and PM were used for comparison with $\mathrm{PC}$, and the results are plotted across frequency in Fig. 9. The claims made from the anechoic simulations are largely seen to be substantiated by the measurements: PC produced ACC-like contrast, ACClike effort and PM-like planarity.

In the anechoic domain, ACC and PC both reached the maximum contrast of $76 \mathrm{~dB}$. In the measured system, the contrast values are more realistic. There is little difference between the measured contrast values, although at frequencies below $600 \mathrm{~Hz}$ the ACC contrast is superior by up to 5 $\mathrm{dB}$. The PM contrast values are similarly degraded, and are the worst among the methods at all frequencies. Although the contrast values begin to fall as frequency decreases, the contrast bandwidth is similar for ACC and PC (dropping below $20 \mathrm{~dB}$ at $3 \mathrm{kHz}$ ), with PM dropping below $20 \mathrm{~dB}$ at 2 $\mathrm{kHz}$. Due to the effects of spatial sampling on the loudspeaker and microphone arrays, the contrast measured on our experimental system continues to reduce above $6 \mathrm{kHz}$. However, this does not affect the usefulness of the optimization cost functions, which can be used for full bandwidth systems when applied to closely spaced line arrays. ${ }^{12,30}$

The control effort performance was comparable between PC and ACC for these filters. The slight increase in control effort for $\mathrm{PC}$ with respect to $\mathrm{ACC}$, noted in the anechoic simulations, is again visible. The $\mathrm{PC}$ control effort was lower than that of PM for all considered frequencies.

Analysis of the measured planarity revealed that below $800 \mathrm{~Hz}$ there was generally more difference between the planarity of PC and PM than in the anechoic case. Nevertheless, 

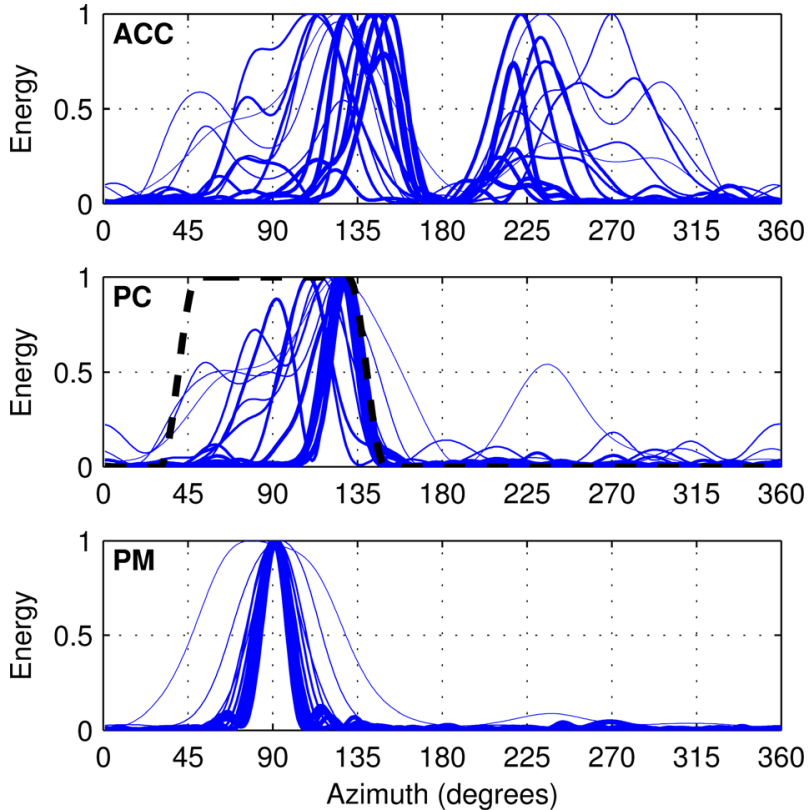

FIG. 10. (Color online) Measured energy across azimuth, analyzed using the planarity beamformer, for ACC (top), PC (middle), and PM (bottom). The thick dashed line in the middle plot indicates the specified window along the diagonal of $\boldsymbol{\Gamma}$. Each line represents a single frequency, with lines at $200 \mathrm{~Hz}$ intervals between 300 and $3000 \mathrm{~Hz}$ superimposed on each plot. Line thickness increases with frequency.

PC represents a significant improvement over ACC in terms of the planarity yielded, over a large part of the frequency range. One region where the measured PC planarity did not perform as highly as expected was between 600 and $800 \mathrm{~Hz}$. The measured energy analysis, shown in Fig. 10, is useful in interpreting this fall in the planarity score. First, it can be noted that the principal components of energy were placed, at each frequency, within the target range of azimuths. However, for some of the curves there is not a single distinct peak, lowering the planarity score. It may therefore be that the window should be narrower to ensure that the energy components arrive from a more consistent direction, while allowing some freedom to ensure that the benefits of $\mathrm{PC}$ at high frequencies are maintained. Perceptual input on the appropriate range of pass angles should also be taken in to account. The measured energy distributions for ACC and PM support the conclusions drawn under free-field conditions.

The performance in the target zone may be assessed by visualizing the sound field. Figure 11 shows the real part of complex pressure at $3 \mathrm{kHz}$, for the simulated results (Sec. V) and the measured pressures (used for Figs. 9 and 10). Comparison between the simulated and measured sound fields reveals that there is increased level variation in the measured case, which is not surprising given the practical considerations of calibration error, RIR variability and measurement noise. Importantly, the main properties of the methods are seen to hold for the measured data: ACC produced a checkered wave field with a notch in the center, PC produced an approximately planar wave field, and PM produced a close approximation to a plane wave from the expected direction with least level variation. The sound field properties expected from the simulated results were thus validated by the performance measurements.
ACC
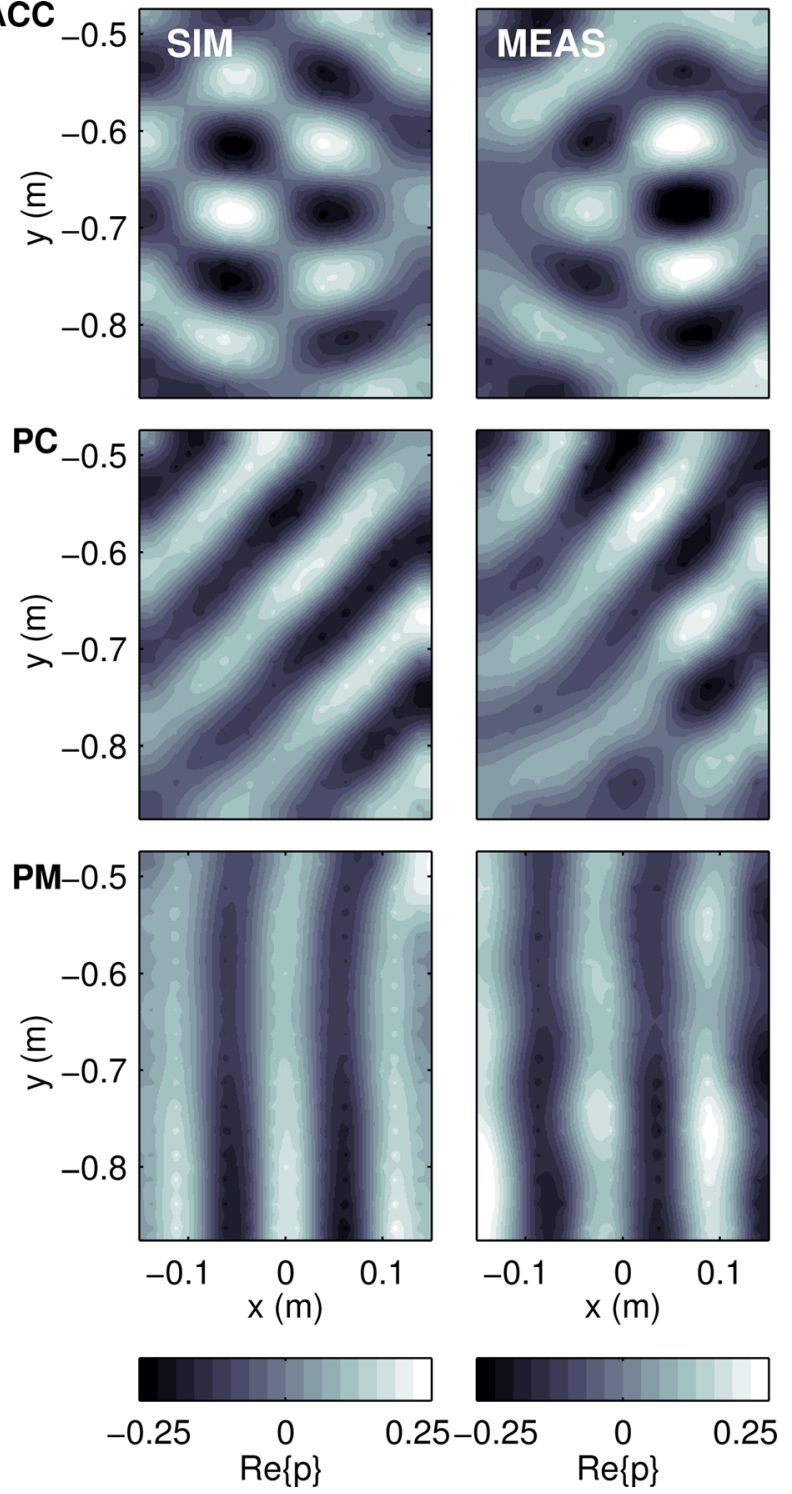

FIG. 11. (Color online) Real part of the complex sound pressure for simulated (left column) and measured (right) target zones at $3 \mathrm{kHz}$ using ACC (top row), PC (middle), and PM (bottom).

\section{DISCUSSION}

PC performed well in anechoic simulations and was shown to produce ACC-like acoustic contrast and control effort, and PM-like planarity. Similarly, the measured performance of PC validated the method's significance for sound zone reproduction. PC did not perform best under any metric, however it avoided poor scores under all metrics, achieving much better contrast than PM and much higher planarity than ACC. The freedom to design $\Gamma$ is thus a significant benefit of PC, where a wider pass range can be specified for monophonic reproduction, eliminating the selfcancellation patterns of ACC yet allowing freedom for good cancellation compared to PM. Validation results measured in an acoustically treated room supported the conclusions drawn from the anechoic simulations.

The mean scores under each metric are summarized in Table I under both anechoic and measured conditions. These scores demonstrate that although PC does not attain the best 
TABLE I. Mean anechoic and measured performance of ACC, PC, and PM, averaged over $300-6000 \mathrm{~Hz}$. The best two results under each metric are indicated in bold type.

\begin{tabular}{lcccccc}
\hline \hline & \multicolumn{3}{c}{ Anechoic } & \multicolumn{3}{c}{ Measured } \\
\hline & ACC & PC & PM & ACC & PC & PM \\
Contrast (dB) & $\mathbf{7 5 . 7}$ & $\mathbf{7 5 . 8}$ & 28.2 & $\mathbf{2 0 . 8}$ & $\mathbf{1 9 . 9}$ & 11.4 \\
Effort (dB) & $\mathbf{- 8 . 4}$ & $\mathbf{- 6 . 9}$ & -0.7 & $\mathbf{- 7 . 3}$ & $\mathbf{- 5 . 6}$ & -2.9 \\
Planarity (\%) & 68.7 & $\mathbf{9 6 . 6}$ & $\mathbf{9 7 . 7}$ & 65.1 & $\mathbf{8 4 . 7}$ & $\mathbf{9 0 . 5}$ \\
\hline \hline
\end{tabular}

scores under each metric, it successfully combines the desirable properties of the other state of the art methods. ${ }^{3}$ In particular, PC reproduced sound fields with significantly simpler distributions of bright zone energy than ACC, with energy components being placed at a range of azimuths within the user-specified pass region. Furthermore, consistently high levels of acoustic contrast were maintained well above the loudspeaker array spatial aliasing limit, when a relaxed constraint was placed on the directions of the incoming plane wave energy. Such a pass window can be specified for monophonic reproduction and will remove the standing wave artifacts from the bright zone pressure distribution over azimuth.

The perceptual experience of the sound zone optimization approaches described in this article can only be fully determined by listening tests. However, the contrast values do give some indication of the effect of interference based on previous perceptual work. Druyvesteyn \& Aarts ${ }^{1}$ found that a minimum contrast of $11 \mathrm{~dB}$ was required, with around $20 \mathrm{~dB}$ preferable. More recent work $^{33,34}$ has found that $10-30 \mathrm{~dB}$ of contrast is necessary for the interference to be perceived as "acceptable," with factors such as the task and program item influencing the listener judgments. Thus, the mean acoustic contrast of $20 \mathrm{~dB}$ measured in our system for PC indicates that there is some practical use for the method. Listening tests are also necessary to determine whether the design of a broad angular pass-range is useful in terms of the listeners localization of the sound source.

In addition to the perceived interference and spatial properties of the methods, the sound zone processing has some effect on the basic audio quality of the target program. In particular, pre-echoes can be present due to the complexity of the filters. These artifacts have been shown to be less severe for PM compared to ACC under anechoic conditions, ${ }^{11}$ but they can be problematic for all methods when using measured RIRs in a reflective room. The effect of pre-echoes can be reduced by pre-processing of RIRs and increased regularization. ${ }^{35}$ This suggests that a trade-off may exist between the achieved contrast and the target quality, and this would also be an interesting topic for perceptual experiments.

\section{SUMMARY}

Planarity control has been proposed as an optimization cost function that produces a planar target zone, as well as producing significant cancellation between zones. Anechoic simulations showed the method to perform similarly to the baseline methods of acoustic contrast control in terms of acoustic contrast and pressure matching in terms of planarity. Measured data, recorded via independent measurements in a reflective room, showed the ranking among these methods to be retained, with PC producing $12-30 \mathrm{~dB}$ of acoustic contrast over the range $300 \mathrm{~Hz}$ to $6 \mathrm{kHz}$. The perceptual effect of sound energy impinging from various angles over frequency (in the context of a personal audio system with interfering audio) should be evaluated in further work.

\section{ACKNOWLEDGMENTS}

This research was supported by Bang \& Olufsen A/S and an EPSRC DTG, and forms part of the POSZ project. Thanks to Alice Duque for assistance with the experimental work, and to Martin Møller, Martin Olsen, Adrian Celestinos and Søren Bech for useful discussions.

${ }^{1}$ W. F. Druyvesteyn, R. Aarts, A. Asbury, P. Gelat, and A. Ruxton, "Personal sound," Proc. Inst. Acoust. 16, 571-585 (1994).

${ }^{2}$ W. F. Druyvesteyn and J. Garas, "Personal sound," J. Audio Eng. Soc. 45, 685-701 (1997).

${ }^{3}$ P. Coleman, P. J. B. Jackson, M. Olik, M. Møller, M. Olsen, and J. A. Pedersen, "Acoustic contrast, planarity and robustness of sound zone methods using a circular loudspeaker array," J. Acoust. Soc. Am. 135, 1929-1940 (2014).

${ }^{4}$ Y. Wu and T. Abhayapala, "Spatial multizone soundfield reproduction: Theory and design," IEEE Trans. Audio Speech Lang. Proc. 19, $1711-1720$ (2011).

${ }^{5}$ A. J. Berkhout, D. de. Vries, and P. Vogel, "Acoustic control by wave field synthesis," J. Acoust. Soc. Am. 93, 2764-2778 (1993).

${ }^{6} \mathrm{D}$. Ward and T. Abhayapala, "Reproduction of a plane-wave sound field using an array of loudspeakers," IEEE Trans. Speech Audio Proc. 9, 697-707 (2001).

${ }^{7}$ K. Helwani, S. Spors, and H. Buchner, "The synthesis of sound figures," Multidimens. Syst. Signal Proc. 25, 379-403 (2014).

${ }^{8}$ M. Poletti, "An investigation of 2-D multizone surround sound systems," in Proceedings of the 125th AES Convention, San Francisco, CA (October 2-5, 2008).

${ }^{9} \mathrm{~N}$. Radmanesh and I. S. Burnett, "Generation of isolated wideband sound fields using a combined two-stage Lasso-LS algorithm," IEEE Trans. Audio Speech Lang. Proc. 21, 378-387 (2013).

${ }^{10} \mathrm{~S}$. Spors, H. Wierstorf, A. Raake, F. Melchior, M. Frank, and F. Zotter, "Spatial sound with loudspeakers and its perception: A review of the current state," Proc. IEEE 101, 1920-1938 (2013).

${ }^{11}$ M. F. Simón Gálvez, S. J. Elliott, and J. Cheer, "A superdirective array of phase shift sources," J. Acoust. Soc. Am. 132, 746-756 (2012).

${ }^{12}$ J. Cheer, S. J. Elliott, and M. F. Simón Gálvez, "Design and implementation of a car cabin personal audio system," J. Audio Eng. Soc. 61, 412-424 (2013).

${ }^{13}$ J. Choi and Y. Kim, "Generation of an acoustically bright zone with an illuminated region using multiple sources," J. Acoust. Soc. Am. 111, 1695-1700 (2002).

${ }^{14}$ J.-H. Chang, C.-H. Lee, J.-Y. Park, and Y.-H. Kim, "A realization of sound focused personal audio system using acoustic contrast control," J. Acoust. Soc. Am. 125, 2091-2097 (2009).

${ }^{15}$ J.-H. Chang, J.-Y. Park, and Y.-H. Kim, "Scattering effect on the sound focused personal audio system," J. Acoust. Soc. Am. 125, 3060-3066 (2009).

${ }^{16}$ S. J. Elliot and M. Jones, "An active headrest for personal audio," J. Acoust. Soc. Am. 119, 2702-2709 (2006).

${ }^{17}$ M. Jones and S. J. Elliott, "Personal audio with multiple dark zones" J. Acoust. Soc. Am. 124, 3497-3506 (2008).

${ }^{18}$ S. J. Elliott, J. Cheer, H. Murfet, and K. R. Holland, "Minimally radiating sources for personal audio," J. Acoust. Soc. Am. 128, 1721-1728 (2010).

${ }^{19}$ S. J. Elliott, J. Cheer, J.-W. Choi, and Y. Kim, "Robustness and regularization of personal audio systems," IEEE Trans. Audio Speech Lang. Proc. 20, 2123-2133 (2012).

${ }^{20}$ J. Cheer, S. J. Elliott, Y. Kim, and J.-W. Choi, "Practical implementation of personal audio in a mobile device," J. Audio Eng. Soc. 61, 290-300 (2013).

${ }^{21}$ M. Shin, S. Q. Lee, F. M. Fazi, P. A. Nelson, D. Kim, S. Wang, K. H. Park, and J. Seo, "Maximization of acoustic energy difference between two spaces," J. Acoust. Soc. Am. 128, 121-131 (2010). 
${ }^{22}$ M. Olik, J. Francombe, P. J. B. Jackson, P. Coleman, M. Olsen, M. Møller, R. Mason, and S. Bech, "A comparative performance study of sound zoning methods in a reflective environment," in Proceedings of the 52nd AES International Conference, Guildford, UK (September 2-4, 2013).

${ }^{23}$ J.-H. Chang and F. Jacobsen, "Sound field control with a circular doublelayer array of loudspeakers," J. Acoust. Soc. Am. 131, 4518-4525 (2012).

${ }^{24} \mathrm{~T}$. Betlehem and P. Teal, "A constrained optimization approach for multizone surround sound," in IEEE International Conference on Acoustics, Speech Signal Processing (ICASSP), Prague (May 22-27, 2011), pp. 437-440.

${ }^{25}$ Y. Cai, M. Wu, and J. Yang, "Sound reproduction in personal audio systems using the least squares approach with acoustic contrast control constraint," J. Acoust. Soc. Am. 135, 734-741 (2014).

${ }^{26}$ P. Jackson, F. Jacobsen, P. Coleman, and J. Pedersen, "Sound field planarity characterized by superdirective beamforming," Proc. Meet. Acoust. 19, 055056 (2013), presented at ICA2013, Montreal (June 2-7, 2013).

${ }^{27}$ J.-W. Choi and Y.-H. Kim, "Manipulation of sound intensity within a selected region using multiple sources," J. Acoust. Soc. Am. 116, $843-852$ (2004)

${ }^{28}$ J.-H. Chang, J.-W. Choi, and Y.-H. Kim, "A plane wave generation method by wave number domain point focusing," J. Acoust. Soc. Am. 128, 2758-2767 (2010).
${ }^{29}$ P. Coleman, P. J. B. Jackson, M. Olik, and J. A. Pedersen, "Optimizing the planarity of sound zones," in Proceedings of the 52nd AES International Conference, Guildford, UK (September 2-4, 2013).

${ }^{30}$ M. F. Simón Gálvez, S. J. Elliott, and J. Cheer, "The effect of reverberation on personal audio devices," J. Acoust. Soc. Am. 135, 2654-2663 (2014).

${ }^{31}$ B. D. V. Veen and K. M. Buckley, "Beamforming: A versatile approach to spatial filtering," IEEE ASSP Mag. 5, 4-24 (1988).

${ }^{32}$ J.-H. Chang, J.-W. Choi, and Y.-H. Kim, "Reproduction of a plane-wave sound field based on wave number domain focusing: Comparison with ambisonics and WFS," in 8th International Conference on Motion and Vibration Control, Daejeon, Korea (August 27-30, 2006).

${ }^{33}$ J. Francombe, R. Mason, M. Dewhirst, and S. Bech, "Determining the threshold of acceptability for an interfering audio programme," in Proceedings of the 132nd AES Convention, Budapest, Hungary (April 26-29, 2012).

${ }^{34} \mathrm{~K}$. Baykaner, C. Hummersone, R. Mason, and S. Bech, "The prediction of the acceptability of auditory interference based on audibility," in Proceedings of the 52nd AES International Conference, Guildford, UK (September 2-4, 2013).

${ }^{35}$ S. G. Norcross, G. A. Soulodre, and M. C. Lavoie, "Subjective investigations of inverse filtering,” J. Audio Eng. Soc. 52, 1003-1028 (2004). 